Canadian University Music Review

Revue de musique des universités canadiennes

\title{
Glenn Gould : Le dernier puritain, écrits 1, réunis, traduits et présentés par Bruno Monsaingeon. Paris : Fayard, 1983, 285 pp.
}

\section{Ghyslaine Guertin-Bélanger}

Numéro 5, 1984

URI : https://id.erudit.org/iderudit/1014019ar

DOI : https://doi.org/10.7202/1014019ar

Aller au sommaire du numéro

Éditeur(s)

Canadian University Music Society / Société de musique des universités

canadiennes

ISSN

0710-0353 (imprimé)

2291-2436 (numérique)

Découvrir la revue

Citer ce compte rendu

Guertin-Bélanger, G. (1984). Compte rendu de [Glenn Gould : Le dernier puritain, écrits 1, réunis, traduits et présentés par Bruno Monsaingeon. Paris :

Fayard, 1983, 285 pp.] Canadian University Music Review / Revue de musique des universités canadiennes, (5), 351-357. https://doi.org/10.7202/1014019ar

All Rights Reserved (c Canadian University Music Society / Société de musique des universités canadiennes, 1984
Ce document est protégé par la loi sur le droit d'auteur. L'utilisation des services d'Érudit (y compris la reproduction) est assujettie à sa politique d'utilisation que vous pouvez consulter en ligne.

https://apropos.erudit.org/fr/usagers/politique-dutilisation/ 
notre pays, en toute occasion, un brevet d'infériorité, en présentant, sans autre forme d'examen, des générations entières de compositeurs comme de simples épigones des grandes figures européennes. Ne serait-il pas plus instructif et honnête en cette matière de donner à l'oeuvre musicale la place centrale qui lui revient? L'EMC elle-même tirerait avantage de cette orientation en corrigeant l'impression que la version actuelle peut laisser d'avoir été écrite davantage par des littéraires que par des musiciens.

Lucien Poirier

Glenn Gould : Le dernier puritain, écrits 1, réunis, traduits et présentés par Bruno Monsaingeon. Paris : Fayard, 1983, 285 pp.

Premier volet d'une "intégrale " des écrits de Gould, cet ouvrage cède la parole au "moraliste austère " dont l'activité réflexive, inséparable de la pratique musicale, revêt la forme d'essais philosophiques, d'essais critiques et d'entrevues fictives. Gould expose sa conception de la technologie et de l'enregistrement, sa philosophie de la communication axée sur la recherche d'une production et d'une jouissance créatrices essentiellement musicales. Privilégiant la signification intrinsèque de l'œuvre musicale, Gould rêve d'une présence sans faille qui, tant du côté du compositeur, de l'interprète que de l'auditeur, échappe à la sphère de l'informe, de l'extériorité, du visuel. Refus donc de la musique qui se donne en spectacle, de tout élément théâtral et de toute autre forme d'expression éphémère susceptible d'altérer l'existence de la musique dans la musique. Ce désir de pureté, cette exigence de perfection constituent l'univers du discours de Gould qui s'est lui-même décrit comme " le dernier puritain " (p. 43). Bruno Monsaingeon, collaborateur intime de Gould, lui rend un fidèle hommage en présentant cette autre dimension de l'activité créatrice du musicien qu'il a choisi d'organiser en quatre parties: les idées philosophiques de Gould composent la première partie; la seconde porte sur les compositeurs; la troisième traite des interprètes, et la quatrième regroupe ses essais critiques.

La première partie, philosophique, rassemble cinq textes distincts. La décision de Gould de mettre fin, dès 1964, à sa carrière de concertiste repose sur la conviction que la technologie de l'enregistrement modifiera la nature de la composition musicale, de l'interprétation, de l'écoute, et conduira à un nouveau type de communication avec l'œuvre musicale. Lors même de ses premières expériences radiophoniques, en tant qu'interprète, Gould fait l'apprentissage avec la façon magistrale de la technologie. Il a retenu, dans l'Épître aux Parisiens, l'essentiel de cet enseignement : " J'avais appris, dit-il, à être malhonnête dans un sens créateur " (p. 25). L'utilisation de l'appareillage technique visait en 
effet une seule fin : « (...) donner une idée de ce qui n'était pas» (p. 25). Cette dimension créatrice de la technologie est au centre même du plaidoyer en faveur de l'enregistrement inscrit dans un des textes les plus denses et les plus controversés de Gould, L'enregistrement et ses perspectives. Gould tend à démontrer comment l'enregistrement peut servir la musique elle-même. À cette fin, il se penche à la fois du côté du compositeur, de l'interprète et de l'auditeur.

Gould examine le rôle de l'enregistrement au niveau de la production de l'œuvre musicale, en se référant à Arnold Schoenberg. Ce compositeur a été selon Gould, l'un des premiers à créer de nouvelles combinaisons instrumentales en fonction du potentiel analytique du microphone et de la clarté qui résulte de son utilisation. La Sérénade, op. 28 et le Septuor, op. 29 l'illustreraient de manière exemplaire. Parmi les avantages que présente l'enregistrement, se greffe l'occasion unique pour les compositeurs qui possèdent quelques talents d'exécution, de fixer, "d'éterniser, en la précisant leur notation " (p. 80). Gould souligne à titre d'exemples, les réalisations de Benjamin Britten et se réfère à la grande partie de l'œuvre de Stravinsky exécutée par le compositeur lui-même pour CBS. Toutefois, "le Stravinsky de Stravinsky " ne risque-t-il pas de freiner la liberté créatrice des autres exécutants? En d'autres termes, peut-on penser que la reproduction de l'œuvre musicale soit exposée ainsi à revêtir le sens d'une simple répétition, imitation ou copie de l'original ? Il serait illusoire de croire en la possibilité d'une interprétation parfaitement exacte, fixée pour l'éternité. Le Stravinsky de Bernstein ou encore de Karajan, cité par Gould, trahirait cette différence à l'œuvre dans toute interprétation. L'ouvre musicale est le produit d'une subjectivité, d'une époque, l'exécution qui lui donne vie aussi. Mais qu'en est-il du nouvel exécutant que préconise Gould?

À l'abri de l'imprévu, du risque de la représentation et de la frivolité du succès virtuose, le nouvel exécutant a le privilège d'établir un contact intime avec l'ouvre musicale. L'analysant, la disséquant afin de rechercher son organisation, sa signification. Il jouit, en tant qu'interprète, des "pouvoirs décisionnels " jadis réservés au seul compositeur. Les ressources techniques de l'enregistrement, collage, montage, contribuent à abattre cette cloison qui l'a longtemps séparé du compositeur, et lui fournissent toutes les chances de satisfaire son souci de perfection. La possibilité qui lui est offerte de reprendre plusieurs fois une même pièce, un même mouvement ou une même séquence procure un sentiment de sécurité que Gould considère indispensable à la réalisation de la cohérence du discours musical et de sa pleine intensité. Le nouvel exécutant a le loisir de s'affranchir de la spécialisation qui lie le concertiste à un répertoire spécifique. L'enregistrement lui permet d'explorer des oeuvres moins connues ou encore de s'attaquer à des productions de grande envergure comme les intégrales. Isolé physiquement de son auditeur, par l'appareillage 
technique, le nouvel exécutant cherche néanmoins, selon Gould, à rejoindre cet auditeur à des niveaux purement intellectuels et auditifs. En quoi consiste plus précisément la participation du «nouvel auditeur »?

Loin de se complaire dans un vide sonore, le nouvel exécutant que préconise Gould peut se doter de conditions idéales afin de comprendre la cohérence interne de l'œuvre. Il est en mesure d'exercer à son tour des actes interprétatifs, recréant au moyen de la manipulation de son puissant appareil, sa propre vision idéale de l'ouvre. Gould imagine facilement cet auditeur qui, équipé de techniques de montage, reconstruit une interprétation en empruntant par exemple quelques mesures voulues à la cinquième symphonie de Beethoven, version Klemperer, pour les intégrer dans la version de Bruno Walter (p. 99). Gould réclame un auditeur-artiste qu'il situe au niveau d'un monde contemplatif et extatique appartenant à la fois au compositeur et à l'interprète. Tous trois sont reliés par la recherche de la cohérence de l'ouvre et par la saisie de la beauté du moment. Une telle participation de l'auditeur est, selon Gould, irréalisable au concert, principalement à cause de "la position de pouvoir et de domination " (p. 32) que détient l'exécutant par rapport à l'auditeur. C'est du moins ce qu'il affirme dans l'entrevue fictive Glenn Gould interviewe Glenn Gould au sujet de Glenn Gould qui mérite d'être soulignée à cause de l'originalité de la forme et du contenu. Le lecteur reconnaîtra les principaux traits de caractère du moraliste qui ont aussi contribué à alimenter son personnage mythique.

Le plaidoyer de Gould en faveur de l'enregistrement converge vers la conclusion suivante : "L'enregistrement et les médias en général représentent l'avenir (...) Inversement, la salle de concert, de récital ou d'opéra représente le passé (...), le passé de la musique " (p. 30). Les arguments de Gould ne sont pas toutefois sans provoquer de nombreuses oppositions de la part des tenants de la salle de concert. Gould les a prévenues en mettant en exergue à son texte (L'enregistrement et ses perspectives), les thèses et antithèses des maîtres qui se sont penchés sur les différents problèmes que pose l'enregistrement, laissant au lecteur le plaisir d'en opérer sa propre synthèse. En présentant ainsi d'autres avenues possibles, Gould provoque, stimule l'autonomie du lecteur plutôt que de l'installer dans un confort dogmatique. Car pour Gould, l'indépendance de la pensée constitue le fondement même de toute créativité : efforcez-vous "d'exister pour et par vous-mêmes " (p. 51), affirme-t-il dans un discours prononcé à l'occasion d'une remise de diplômes de fin d'année au conservatoire de Toronto. Et s'il avait été invité un jour à rencontrer les étudiants de la Julliard School, il les aurait incités à développer la même attitude dans leur rapport à la partition : "Essayez tout au moins de vous faire une idée à vous de chaque partition et n'hésitez pas à vous y conformer " (p. 110). L'essentiel réside pour Gould dans la recherche du sens de l'ouvre à interpréter, axée sur 
une démarche davantage analytique que mécaniste. Au-delà des considérations proprement techniques-pianistiques, le travail de "l'interprète-compositeur " consiste à sculpter les formes, à organiser la structure de l'œuvre et à l'exprimer avec le plus de clarté et de netteté possible. Nul besoin, pour y parvenir, d'être esclave de son instrument. Le musicien devrait plutôt apprendre à développer sa "mémoire analytique " et "sa concentration ». Les conseils que nous livre Gould au sujet de l'apprentissage pianistique, à partir de sa propre expérience, dans le chapitre Glenn Gould et le piano qui clôt la première partie de l'ouvrage, ne manquent pas de pertinence même s'ils sont teintés d'irréalisme. Qu'en est-il de l'étudiant qui ne possède pas la facilité technique de Gould? Le virtuose ne lui réserve aucune recette. La seule démarche efficace repose, selon Gould, sur la capacité du cerveau à assimiler les problèmes pratiques qui se posent tout au long de l'analyse d'une partition qui évidemment doit être exempte de tout doigté.

Le seconde partie de l'ouvrage est consacrée aux créateurs de formes. Une attention particulière est accordée entre autres aux compositeurs Richard Strauss et Arnold Schoenberg. L'analyse qui leur est destinée repose sur un principe qui laisse entendre de nouveau le leitmotiv caractéristique de la démarche de Gould : l'œuvre elle-même, sa forme, son organisation harmonique, rythmique, mélodique. Gould insiste sur le fait que la nouveauté musicale n'est pas nécessairement synonyme de grand art ni de progrès. De même la position historique d'une œuvre ne doit pas être un facteur déterminant pour son évaluation. Gould ne nie pas la nécessité de se pencher sur les conditions historiques qui ont présidé à la naissance de l'œuvre, de la forme qu'elle revêt. Mais cette démarche doit converger vers la compréhension de la dimension matérielle de l'œuvre. Gould précise sa pensée en abordant Richard Strauss comme « la plus grande personnalité musicale du vingtième siècle " (p. 177), même s'il fut " à contre courant de la mode " (p. 188). Gould fait remarquer que son "évolution stylistique » peut être qualifiée "de rétrograde, historiquement parlant» (p. 188). Toutefois sa valeur résiderait dans la "cohérence extraordinaire de son vocabulaire » (p. 179) harmonique, dans la qualité de son contrepoint, même s'il ne fut en aucune manière un réel contrapuntiste. La conclusion de Gould, au sujet de ce compositeur, riche en enseignement, mérite d'être retenue : « La grandeur de la musique de Strauss (...) fournit l'exemple d'un homme qui enrichit son temps en n'y appartenant pas; qui parle pour toutes les générations en n'appartenant à aucune » (p. 186). À l'inverse de Strauss, Schoenberg, "ce meneur d'avantgarde " représenterait, auprès de certains mélomanes, "la plus importante figure du siècle » et ce, principalement, en tant que théoricien. Or selon Gould, il est plus facile de prouver l'importance qu'a eue Schoenberg pour notre époque que de démontrer la grandeur et la profondeur de son ouvre musicale. C'est ce dernier objectif que poursuit Gould en passant par la description des étapes fascinantes de la chronologie du compositeur. 
Un autre créateur de formes, J.S. Bach, prend place à côté des deux compositeurs précédents. Gould justifie son admiration et son enthousiasme pour ce musicien en établissant une distinction entre "musique inductive » et "musique déductive». Les fugues de Bach appartiendraient à la catégorie de "musique inductive (...) dont la structure formelle s'identifie avec l'évolution d'une idée ou d'un complexe d'idées thématiques spécifiques" (p. 153). Ainsi toute la richesse d'une fugue réside selon Gould dans le fait qu'elle soit " une invitation à inventer une forme" (p. 126). L'Art de la fugue que Gould a interprété au piano tout au long de sa carrière de concertiste, et enregistré à l'orgue sous l'étiquette Columbia, illustre parfaitement l'aventure créatrice qui revient à l'interprète. Cette ouvre, comme d'ailleurs l'ensemble des fugues de Bach qui ont été écrites pour diverses combinaisons instrumentales, fournit à l'interprète l'occasion d'une "communication re-créatrice». Gould, au moyen d'exemples musicaux, démontre la richesse du matériel harmonique, l'originalité des thèmes, la variété des modulations.

À l'inverse de cette "musique inductive » que Gould affectionne, la "musique déductive» serait celle "dans laquelle on force les matériaux utilisés à rentrer dans le cadre d'un schéma formel préalablement établi » (p. 153). L'Allegro d'une sonate appartiendrait à cette catégorie musicale avec laquelle Gould ne partage aucune affinité. C'est le même sentiment qu'il entretient à l'égard de Mozart. On connaît les propos provocateurs au sujet de ce compositeur que Gould a déjà communiqués à Jonathan Cott, lors d'une entrevue qu'il lui accordait en 1974. Bruno Monsaingeon, en bon mozartien, rencontrait Gould en 1976, afin d'appronfondir cette vision bien personnelle de Mozart. On y retrace les mêmes affirmations que dans les entretiens avec Cott : "Mozart est devenu un compositeur médiocre, (...) il est mort trop vieux " (p. 137). Gould récuse en effet la théâtralité dans l'œuvre du compositeur grandissant. Par ailleurs, il avoue une préférence marquée pour les sonates de jeunesse " aux vertus baroques " qui contribuent à satisfaire son appétit contrapuntique. Gould affirme prendre plaisir à les analyser, à les disséquer, à les interpréter en altérant parfois la conduite des voix, en arpégeant les accords, en choisissant son tempo selon sa propre pulsation cardiaque ...

Les idées qui accompagnent la description de son héros, Léopold Stokowski, dans la troisième partie de l'ouvrage consacrée aux portraits des interprètes qui l'ont fasciné, permettent de comprendre le problème de la fidélité au compositeur que soulève l'interprétation de Gould. À l'inverse d'un Toscanini que l'on disait "littéral " par son respect aux " desiderata " inscrits dans la partition du compositeur, Stokowski préconisait l'indépendance de l'interprète par rapport au texte du compositeur. Ce musicien, selon Gould, a redéfini le rôle de l'interprète en précisant l'initiative créatrice qui lui revient. L'interprète aurait l'obligation de se dire, de se déclarer, d'exprimer sa propre signification 
de l'œuvre. Stokowski avait déjà compris le rôle de la technologie de l'enregistrement. L'interprète pouvait désormais rechercher une forme plus satisfaisante et se "concentrer sur une vision idéale ». Gould démontre avec son esprit de finesse l'admiration qu'il a eue pour ce musicien qui lui a communiqué le sens de l'extase. Tout en privilégiant la solitude du studio d'enregistrement et le nouveau rôle de l'interprète défini à la manière de Stokowski, Gould ne demeure pas moins fasciné par les musiciens comme Yehudi Menuhin et Arthur Rubinstein dont la vie entière fut consacrée à la carrière de concertiste. Menuhin appartiendrait toutefois à une catégorie bien particulière de musiciens, si l'on se réfère au texte dans lequel Gould lui rend un témoignage émouvant. Il n'hésite pas à le définir selon la manière toute puritaine de concevoir l'artiste en général, comme "missionnaire et héraut ». Il évoque avec nostalgie l'admiration qu'aurait eue sa grand-mère à l'égard d'un musicien qui, sans aucun doute, rejoignait sa définition de l'artiste porte-parole.

Par ailleurs, Gould ne cache pas son désaccord avec les idées du pianiste Rubinstein. Le débat opposant salle de concert et studio d'enregistrement refait surface. Rubinstein rejette les techniques de montage, préférant interpréter une ouvre sans interruption au risque d'y percevoir quelques imperfections. Il croit à la communication intense avec l'auditeur de la salle de concert. Pour sa part, Gould réaffirme sa définition de l'interprète-compositeur " grâce à l'enregistrement et poursuit une réflexion d'ordre moral sur le rôle de la technologie. S'inspirant du théologien québécois Jean Le Moyne, Gould définit la technologie comme étant " une entreprise charitable " (p. 253). Il conçoit l'enregistrement comme solution au système de compétition présent non seulement dans le monde musical mais dans la société en général. Ce thème de la compétition revêt une dimension importante dans la morale de Gould. L'esprit compétitif représenterait « la racine du mal ». C'est à ce titre qu'il rejette la forme du concerto qu'il perçoit comme «l'analogie musicale parfaite» de la compétition.

La réflexion morale apparaît en effet indissociable des préoccupations intellectuelles et musicales de Gould. Geoffrey Payzant, dans son ouvrage traduit récemment en français chez Fayard, Glenn Gould, un homme du futur, s'est appliqué à démontrer en analysant l'ouvre littéraire de Gould, le rapport constant qu'il établit avec la morale lorsqu'il se penche sur la nature et le rôle de la technologie, de l'enregistrement et de l'art en général. L'ouvrage du professeur Payzant constitue un outil complémentaire important pour comprendre la littérature gouldienne. S'il retient présentement notre attention, c'est pour souligner non seulement l'intérêt de son contenu mais aussi pour aborder une autre catégorie de l'œuvre littéraire de Gould : les essais critiques. Combinant le sérieux et l'humour, Gould s'est livré à l'exercice de la recension du Gould de Payzant. Il reconnait l'objectivité académique impeccable de Payzant, "son expérience de l'esthétique " 
et « sa connaissance pratique de la musique » qu'il a su combiner pour comprendre la pensée musicale de Gould.

Les biographes ne possèdent pas tous cette qualité d'objectivité que Gould attribue à Payzant. Dans son essai critique, Boulez-le-terrible, Gould dénonce l'ouvrage de Joan Peyser qui décrit le portrait "psychobiographique " du compositeur Pierre Boulez, en attirant l'attention du lecteur sur les expériences tumultueuses de sa vie, tissée de "complots, de contre-complots, de brouilles, de réconciliations ». Peyser aurait été, selon Gould, "la proie des fantasmes de la mode ». Son ouvrage n'est rien d'autres qu'une pièce dont la moralité serait : " celui qui vit par le glaive périra par le glaive ». Mais, de continuer Gould, on ne saurait douter que «la sympathie de Peyser va au glaive plutôt qu'à sa victime " (p. 282).

Entre ces deux essais critiques, Gould exprime son admiration inébranlable pour Barbara Streisand en procédant à la recension d'un disque, "Classical Barbara », paru en 1976 pour la maison Columbia. Il établit une comparaison entre l'art de cette interprète et celui d'Elizabeth Schwarzkopf. Ses affirmations dithyrambiques à l'égard de Streisand reposent sur une bonne connaissance de la technique vocale de l'interprète et des particularités de son style d'expression. Il emprunte à sa sensibilité et à son sens analytique, les instruments nécessaires pour démontrer que le disque "Classical Barbara " n'est pas vraiment bon ni vraiment mauvais (p. 274).

Si Glenn Gould est "le dernier puritain » (ses écrits nous ont permis de le constater), il est aussi paradoxalement " un homme du futur ». Bien avant qu'il soit question du "virage technologique ", Gould a su comprendre et valoriser la nature et le rôle de la technologie dans le domaine de l'art musical. Sa réflexion est non seulement au centre même des préoccupations socio-culturelles de notre époque, mais elle rejoint par son questionnement une dimension laissée pour compte par les tenants de la modernité. L'ouvre littéraire de Gould constitue un instrument essentiel à la reconstitution de sa " poïétique ", c'est-à-dire de ses intentions esthétiques, philosophiques, des conditions matérielles, psychologiques susceptibles de nous faire comprendre les choix qui l'ont guidé dans son interprétation créatrice. Plus qu'un instrument de travail, les écrits de Gould, par la beauté et l'originalité de l'écriture, invitent le lecteur à découvrir le plaisir du texte.

Ghyslaine Guertin-Bélanger

Glenn Gould, entretiens avec Jonathan Cott, traduit et présenté par Jacques Drillon. Paris : Lattès, Collection Musiques et Musiciens, 1983, $156 \mathrm{pp}$.

Les entretiens avec le pianiste canadien Glenn Gould réalisés par 